\title{
Measurement of Reflectance Properties of Asphalt using Photographical Methods
}

\author{
Catalin Daniel Galatanu \\ Faculty of Civil Engineering \\ Gheorghe Asachi Technical University \\ Iasi, Romania \\ catalin.galatanu@tuiasi.ro
}

\author{
Laurent Canale \\ IEEE Senior Member \\ LAPLACE Laboratory; UPS; INPT; CNRS \\ Toulouse University \\ Toulouse, France \\ laurent.canale@laplace.univ-tlse.fr
}

\begin{abstract}
Measurement of reflectance properties of asphalt is an insufficiently addressed problem, although this parameter is involved in three major directions in which the development of roads and lighting takes place: new technologies for materials, including solar energy harvesting, energy efficiency of road lighting and light pollution reduction. For each of these directions, concerns for improving performance have been identified, in which for each percentage gained, significant efforts and costs are registered. It is thus shown that although the reflectance properties of asphalt can change the results to a large extent (even 47\%), this parameter is considered as a material constant and is not measured, although it is variable over time. After evaluating some landmarks regarding the geometric domain for which this parameter must be measured, a photographic method is presented and exemplified by which the desired values can be obtained insitu conditions.
\end{abstract}

Keywords-EN 13201, Reduced Luminance Coefficient, Asphalt albedo, Luminance map, Reflectance uncertainty, street lighting, light pollution

\section{INTRODUCTION}

The paper identified a parameter that underlies the calculation of the luminance of a street lighting system, the reflectance qualities of the asphalt. Usually predetermined data are used, defined for large categories of asphalt (Europe or USA), with few possibilities to estimate the real state of the materials. It is a challenge that while looking for highly accurate results in terms of calculation, for example, the reflectance factor is responsible for a high degree of approximations.

\section{DIFFERENT MOTIFS FOR REFLECTANCE MEASUREMENT}

The problem of asphalt, as a solution for roads, is beginning to be questioned. In this very recent article [1] we are challenged with the vision that the asphalt, as we know it, will be replaced by entirely other material, with various reasons (solar energy with photovoltaic or thermoelectric conversion, reuse of waste material). For these new materials, for which their behavior over time is not known, it is all the more important to measure the reflection factor.

\section{A. Harvesting Solar Energy and Reflectance Factor}

One of the most promising directions of road evolution is the production of electricity through photovoltaic conversion [2]. Obviously, the main objective will be the transparency and durability of the surface, so measuring reflexivity is a need. But there is also the possibility of harvesting solar energy through thermal or thermoelectric conversion [3]. Even if the amplitude of this method is less developed compared with PV conversion, the main objective becomes to obtain as high absorption coefficient as possible (albedo as low as possible), which needles contradict the requirement to obtain high luminances during night time, for good visibility.

In other research concerning global warming and infrared asphalt response [4] one discover the confirmation that the various types of asphalt have responded very differently to each other, depending on composition (structure) and wear or age. An important conclusion in [4] is that "the dependence of the diffuse reflectance factor on the light spot size, in order to determine a minimum light spot size for any further optical study. By comparing the BRF (bidirectional reflectance factor) to a nearly-Lambertian Spectralon ${ }^{\circledR}$ white standard", the conclusions are that the asphalt is in general a good diffuse scatterer. This statement will be contradicted later, and we will confront it with our own measurements.

When a lower IR response is required, the asphalt with high albedo is reached, but it contradicts other very current objectives, such as lane border detection [5]. In this working hypothesis the light color is a disadvantage, because the vision requires a high luminance contrast between asphalt and markings, in order to achieve good visibility.

\section{B. Promoting Energy Efficiency and Reflectance Factor}

Another distinct direction where asphalt is involved is that of energy efficiency of lighting systems. This can be achieved through the light sources themselves (and we have seen the obvious progress of LED sources), but there may be other ways to increase energy efficiency. One of them is dimming, but not in traditional manner. Dimming that follows an original philosophy is presented in [6]. Here it is based on the finding that in the field it is not possible to ensure the constant step between the lighting poles, as is taken into account in the design phase. The distance between poles is almost nonstop reduced by several meters, due to the intersections and the access paths in the properties. These reductions can be offset with custom dim ratio for almost every pole. In relation to this strategy one can propose another factor of dimming, which takes into account that in the project calculations are carried out for new asphalt, so it is a dimming reserve that can be applied after a number of years, along with the wear of the asphalt. For this, however, we need to measure in-situ reflectance, which is also our objective.

There are also classical approaches, where the lighting system is to be modernized [7], but the problem of measuring reflectance factor is neglected, and it is mentioned only:

- Data on poles;

- Geometry arrangements.

Energy efficiency can also be achieved by precise calculations, as in [8], which is a classic and traditional approach. But if there is a purpose of reducing the 
approximations from $20 \%$ to $10 \%$ and then even to $5 \%$ and finally to $1 \%$ it is not allowed to avoid the parameter on which the luminance directly depends, i.e. reflectance factor of asphalt, as seen in [8].

Retrofit solutions can be another approach to energy efficiency. In [9] the optimization of solutions with the help of the computing power of DIALUX is used, but the reflectance answer of asphalt influence is not accessible, nor is it mentioned, which calls into question the entire optimization of [9].

Energy efficiency is also obtained by considering natural lighting during twilight, which changes the time and manner of connecting artificial lighting [10]. After finding that natural lighting is isotropic (after sunset, when the sky is still bright), it reaffirms what was actually known, that artificial lighting is anisotropic, thus contradicting the statement that asphalt is perfectly diffuse, from [4].

\section{Asphalt Reflectance and Light Pollution}

The problem of asphalt reflection can't be dissociated from that of light pollution. Even if light incident on the road is not considered light pollution, what is reflected contributes to light pollution. In [11] is found the exact study of this parameter, the reflective properties of the soil (clear spectral reflection of soil, asphalt, and concrete) and the sky glow.

In [12] this analysis is continued, by extending it to the extreme situation of summer - winter. The crowns (branches) of the trees are also clearly modeled, with or without the presence of leaves or snow. However, the arguments in [12] are also valid for street lighting, without the need for additional arguments.

Optimization of lighting systems can benefit from modern tools, such as genetic algorithm [13]. Here, however, an interesting thing can be found, because the whole optimization refers to the lighting level. Or, if the reflection factor is not considered, then the calculation depending on the lighting can generate oversizing. The approach method is even more surprising when the precise modeling of the light pollution is desired, for which the level of illumination in the areas near the road is evaluated.

There are also fewer approaches, such as [14], where ESCO-type projects are pursued several years after commissioning. It is shown that there are parameters that are not yet in current use. Utilance [15] is one of them, and essentially expresses the ratio between the incident luminous flux on the road and the actual luminous flux emitted by the luminaire. More interestingly, it is emphasized in [14], it is the Installation Lighting Factor, which includes the average luminance of the road and therefore reflectance of the road, with another argument for the necessity of its measurement. Global image of all these factors are presented in Fig.1.

\section{THE COMPLEXITY OF REFLECTANCE MEASUREMENT AND THE STREET LIGHTING PERFORMANCE}

All the arguments so far lead us to the importance of measurement of reflectance factor of the asphalt. In such situations, as those of entrances to road tunnels [16], the contrast of luminances is important to obtain a safe visibility. In these visual scenes, the reflectance of the asphalt is evaluated in the context of the other parameters:

- The brightness of the sky (clear or cloudy);
- Road luminance (day, night);

- Reflectance and luminance of vegetation;

- Emissivity of buildings;

- The luminance of the rocks and the mountainous landscape.

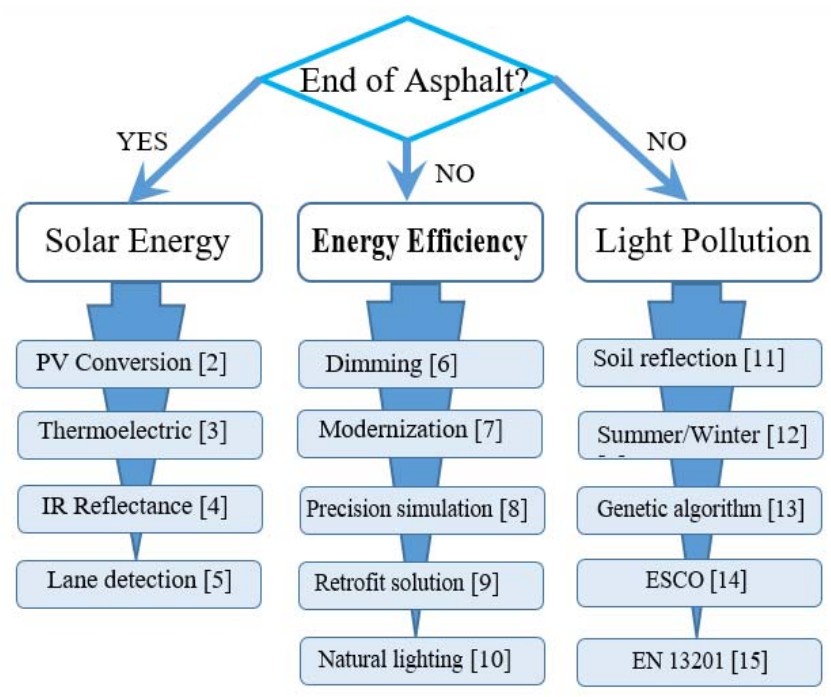

Fig. 1. Influence of reflectance factor of asphalt on other lighting connected aspects.

In contrast to [16] is [17], where there is presented a very complex system of in-situ measurement of road lighting systems, which uses a frame equipped with multiple sensors, a frame carried by a vehicle on the road. Global results of illumination and level of uniformity are obtained, but we do not find any information about luminance. However, a positive example is [18], which demonstrates the importance of analyzing the distribution of luminances, even if only illumination can be accepted for conflict areas [15]. Acceptance of illumination is justified by the fact that in conflict areas (junctions) visibility must be ensured for multiple relative driver-target configurations. The photographic method in [18] demonstrates that global (otherwise unavailable) information can be obtained that includes reflectance of asphalt, including lines or other signs.

However, about the complexity of the problem we are warned in [19], where it is stated that for certain luminance coefficients $q(\beta, r)[15]$, since specular reflection of road surface, "optimal values for luminance and illumination cannot be obtained simultaneously". This contradiction is an additional argument for measuring the reflectance factor of asphalt.

The same parameter acquires another relevance for tropical areas, where high reflectance asphalt is pursued [20]. It is interesting that the implications are analyzed only in the diurnal hypothesis, while the benefits on street lighting could be much more spectacular.

But the actual measurements of these parameters are not simple, as seen in [21], for which some aspects of the experimental program are noted:

- Measurements carried out over three years;

- Nine types of asphalt;

- Two types of wear materials; 
- Asphalt concrete with particles of 11 - 16mm;

- Different ages, between 2 and 10 years;

- Different but moderate wear.

It is obvious that such a systematic approach exceeds the objective of a street lighting project, which needs to be able to achieve an optimal sizing for the situation at a given time.

The final argument for this research is found in the reference work, with monographic character [22], where the specific standards for street lighting are analyzed, in their evolution. It turns out that many of the imposed values are quite arbitrary. There are also interesting statements, namely "in-situ luminance measurement could indicate variations up to $45 \%$ from the design value". The explanation is also provided: "One contribution to this difference is uncertainty in the road surface reflectance data, for example the age and the traffic volumes".

The main situations related to reflectance measurement of the asphalt is presented in Fig.2:

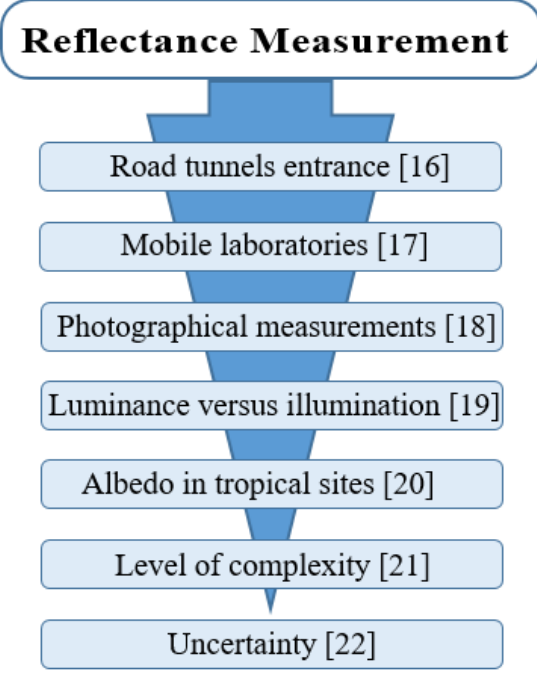

Fig. 2. The problems related to the reflectance measurement of asphalt.

Overall, the uncertainty that exist in many lighting designs due the lack of measurements of asphalt reflectance must be reduced.

\section{REDUCED LUMINANCE COEFFICIENT ASSESSMENT FOR ASPHALT TYPE C2, N1 AND W4}

In order to prepare the measurement program for reduced luminance coefficient $r$ it is necessary to analyze in advance the range of variation of this parameter for the existing data [15], respectively for some types of asphalt. The values are analyzed for the points for which in [15] the calculation of the luminance for an observer located at a height of $1.5 \mathrm{~m}$ and a distance of $60-100 \mathrm{~m}$ is indicated. In Fig.3 are indicated a number of 13 calculation points, distributed with a step of $3 \mathrm{~m}$ along the traffic lane in the vicinity of the poles $(\mathrm{H}=10 \mathrm{~m})$. The values of the angles $\beta$ and $\varepsilon$ in Fig. 3 for the measuring points are indicated in Table 1 . It should be noted that for the following series of measuring points (towards the road axis) these values are very close, which allows us to significantly reduce the number of values for which reduced luminance coefficient $r$ must be measured, without the need to complete the whole value matrix, as indicated in $[15, \mathrm{p} .17]$ for $\operatorname{tg} \beta$ between 0 and 12 and $\varepsilon$ between 0 and $180^{\circ}$.
This greatly limits the number of elements in the matrix with values of reduced luminance coefficient.

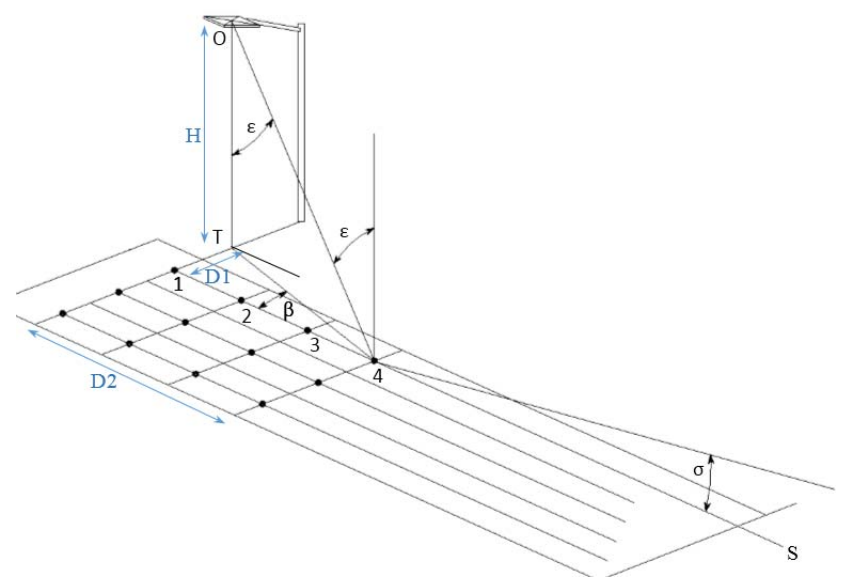

Fig. 3. Points for luminance calculation, according to [15].

In Table 1 it can be seen that after the first 3-4 calculation points near the pillar, the angle $\beta$ reaches $10^{\circ} \div 5^{\circ}$, and the angle $\varepsilon$ reaches $55^{\circ} \div 75^{\circ}$. These areas can reduce the amount of data that needs to be measured and analyzed.

TABLE I. VAlues For ANGLEs Beta AND EPSILON FOT THE POINTS FROM FIG. 3

\begin{tabular}{|c|c|c|c|c|}
\hline & D1 & D2 & $\boldsymbol{\beta}$ & $\boldsymbol{\varepsilon}$ \\
\hline Point no. & $\boldsymbol{m}$ & $\boldsymbol{m}$ & grades & grades \\
\hline 1. & 3 & 0 & 90.00 & 16.70 \\
\hline 2. & 3 & 3 & 45.00 & 22.99 \\
\hline 3. & 3 & 6 & 26.57 & 33.85 \\
\hline 4. & 3 & 9 & 18.43 & 43.49 \\
\hline 5. & 3 & 12 & 14.04 & 51.05 \\
\hline 6. & 3 & 15 & 11.31 & 56.83 \\
\hline 7. & 3 & 18 & 9.46 & 61.28 \\
\hline 8. & 3 & 21 & 8.13 & 64.76 \\
\hline 9. & 3 & 24 & 7.13 & 67.54 \\
\hline 10. & 3 & 27 & 6.34 & 69.79 \\
\hline 11. & 3 & 30 & 5.71 & 71.65 \\
\hline 12. & 3 & 33 & 5.19 & 73.21 \\
\hline 13. & 3 & 36 & 4.76 & 74.53 \\
\hline
\end{tabular}

The next preparatory stage for the measurement program is the estimation of the range of variation of the reflectance factor, for various types of asphalt. In [15] one use another variable, namely reduced luminance coefficient, which is used to calculate the road luminance $L$ for the standard observer, according to [15]:

$$
L=\frac{I \times r \times \emptyset \times M F \times 10^{-4}}{H^{2}}
$$

Where
$I$ - Lighting intensity (cd/klm);
$r$ - Reduced luminance coefficient;
$\varnothing$ - Luminous flux;
$M F$ - Maintenance factor;
$H$ - Mounting height of the luminaire. 
Equation (1) is valid for specular reflection, for which the reduced luminance coefficient $r$ models this reflection. If we accept the hypothesis that the reflection would be perfectly diffuse, then the asphalt would be described with a material with reflectance factor $\rho$ that would produce, for the considered angles, the same luminance $L$ :

$$
L=\frac{\rho \times E}{\pi}
$$

Where $E$ is the illuminance on the calculation point, produced by the same lighting intensity $I$ :

$$
E=\frac{I \times \cos \varepsilon}{l^{2}} \times M F
$$

Where $l$ is the distance between the luminaire and the calculation point.

In (2) one include (3) and we write the equality with (1):

$$
\frac{I \times r \times \emptyset \times M F \times 10^{-4}}{H^{2}}=\frac{\rho}{\pi} \frac{I \times \cos \varepsilon}{l^{2}} \times M F
$$

Making the necessary operation the reflectance factor will be:

$$
\rho=\pi \frac{r \times 10^{-4}}{(\cos \varepsilon)^{3}}
$$

It is necessary to specify that since reduced luminance coefficient $r$ is not constant, neither reflectance $\rho$ will be a constant, but a function of $\beta$ and $\varepsilon$ that is:

$$
\rho(\beta, \varepsilon)=\pi \frac{r(\beta, \varepsilon) \times 10^{-4}}{(\cos \varepsilon)^{3}}
$$

For the first 14 calculation points in the vicinity of the column and asphalt type C2, a variation presented in Fig. 4 is obtained. It is observed that one start from reflection coefficients of $\rho=0,15$ under the column $\left(\varepsilon=0^{\circ}\right)$ and the values increase as the angle of incidence increases to $75^{\circ}-85^{\circ}$.

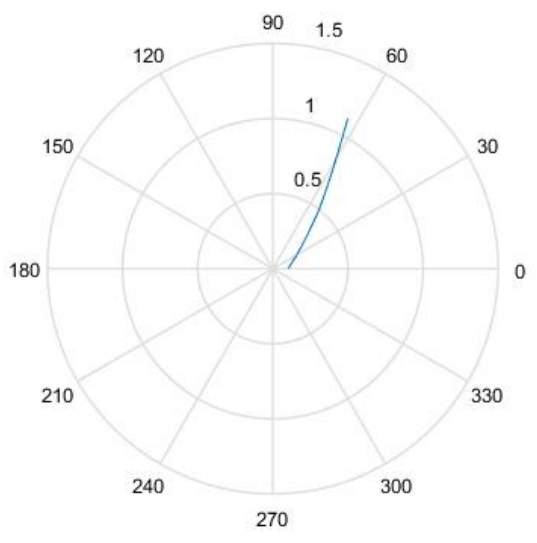

Fig. 4. Reflectance factor in polar coordinates $(\varepsilon)$ for asphalt type C2.

Fig. 4 also forces us to analyze what happens to the reflectance factor for other types of asphalt. In Fig.5 the variation of reflectance factor for $\mathrm{C} 2$ asphalt together with $\mathrm{N} 2$ type asphalt is presented. A different range of variation is observed, but with the same tendency to increase the reflection for large, sharp incident angles. The relative brightness of N2 asphalt is much lower than that of $\mathrm{C} 2$ asphalt. Even if weighted average luminance coefficient $\left(\mathrm{Q}_{0}\right)$ of $\mathrm{C} 2$ Asphalt (CIE) is equal with weighted average luminance coefficient of
$\mathrm{N} 2$ asphalt, respectively $\mathrm{Q}_{0}=0.07$, the $\mathrm{N} 2$ pavement (concrete) is obvious more diffusive.

With this result, the next step is to investigate what happens for the glossiest asphalt, in wet conditions: W4.

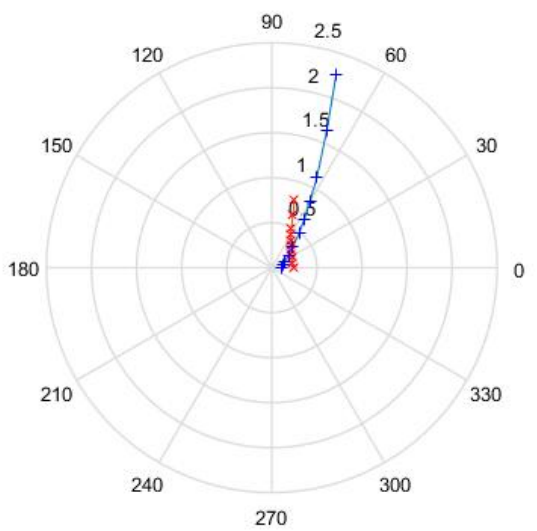

Fig. 5. Reflectance factor in polar coordinates $(\varepsilon)$ for asphalt type C2 (blue and ' + ' and asphalt type N2 (red and ' $x$ ').

The results for reflectance factor for W4 asphalt is presented in Fig.6:

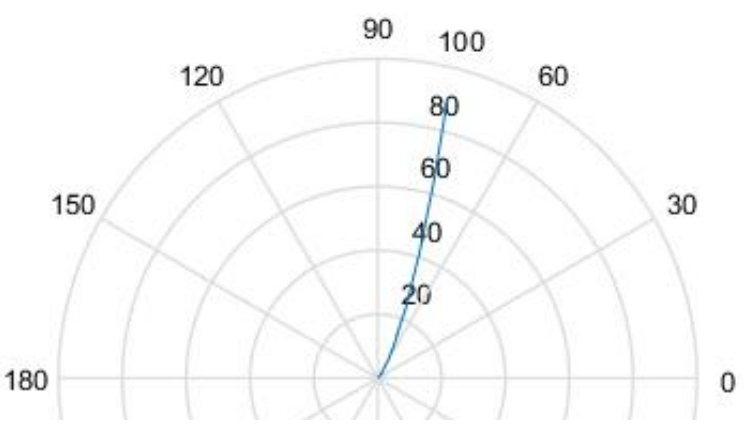

Fig. 6. Reflectance factor in polar coordinates $(\varepsilon)$ for asphalt type W4.

The results shown in Fig. 6 do not allow us to accept that it could be a reflectance factor, being visible an obvious amplification effect. Our conclusion is that the hypotheses that led us to (6) are valid for asphalt with porous structure, and lose their validity for glossy asphalt, and even more so with wet surface. For specular reflection certainly a reflectance factor as (6) does not make sense. These observations are useful in the processing and careful interpretation of data from the reflectance factor measurement.

\section{EXPERIMENTAL MEASUREMENTS}

For the measurement program we started from the sourcepoint geometry of measurement in Fig.3 [15] and with the range of values for $\varepsilon$ and $\beta$ from matrix of the reduced luminance coefficient $r$ [15]. Especially to cover the maximum range for $\operatorname{tg} \varepsilon$, a distance of max. $12 \mathrm{~m}$ of the target point with respect to the optical axis of the light source is

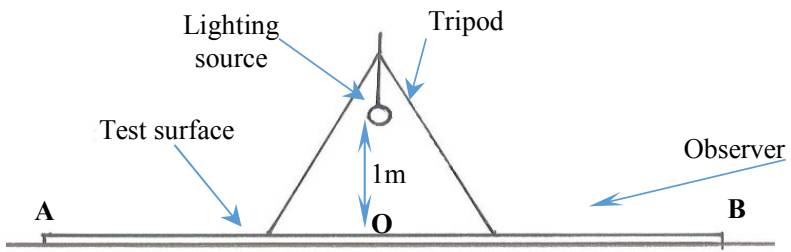

Fig. 7. The mock-up for measurements using imageing method. 
necessary, if lighting source is located at a height of $1 \mathrm{~m}$, a configuration that generates a scale factor of 1:10. To test the measuring principle, a working surface of $5 \mathrm{~m}$ length was used, according to Fig.7. The digital camera calibrated for luminance measurement is placed at a height of $15 \mathrm{~cm}$ and a distance of $6 \mathrm{~m}$, to keep the angles $\sigma$ of the observer as in Fig.3 [15]. For the simplicity of the presentation, we will work only on the observer-light source direction, ie $\beta=0^{\circ}$ and $\beta=180^{\circ}$. The obtained luminance field is presented in Fig. 8:

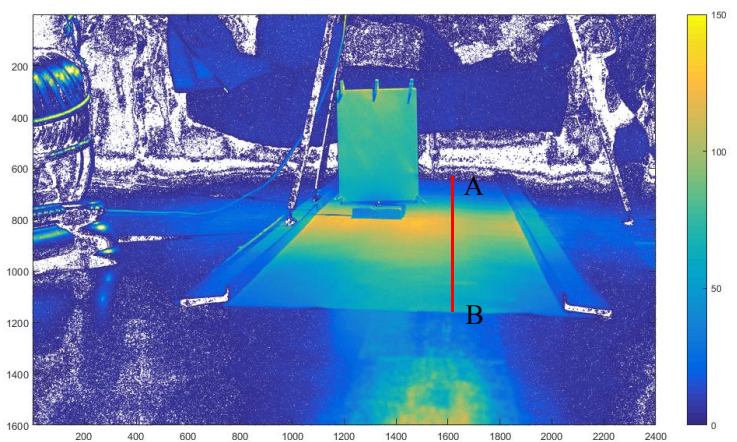

Fig. 8. Luminance map $\left(\mathrm{cd} / \mathrm{m}^{2}\right)$ for the mock-up scene, $A B$ is the measurement domain.

In Fig.8, the $\mathrm{AB}$ line is the measurement domain, corresponding to $\operatorname{tg} \varepsilon=(0$ to 2.5$), \beta=0$ and $\beta=180$. The test surface was a perfect Lambertian material (gray cardboard), with the intention of obtaining a controlled illumination (from a punctiform source), respectively the known incident intensity in each point for which the luminance is measured. The distribution of the illuminations measured and calculated on the $\mathrm{AB}$ direction are available in Fig.9:

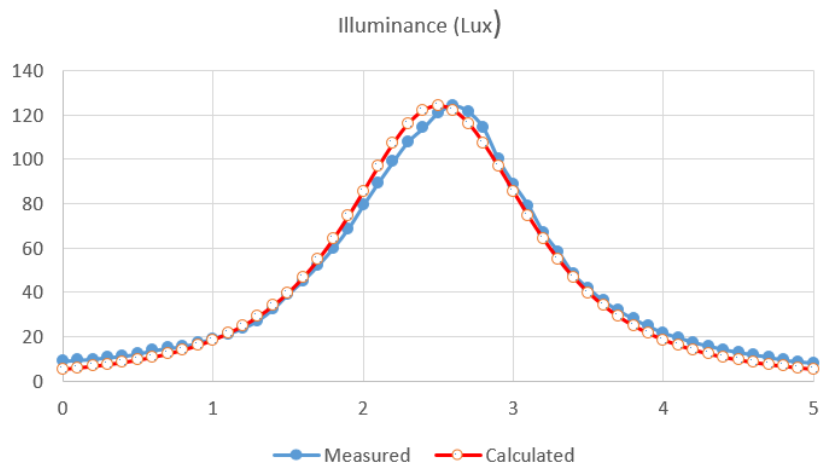

Fig. 9. The illumination level (lux) for the AB direction in Fig.8.

With this known distribution for illumination and therefore for the incident light intensity at each point, it was intended to check the diffuse reflection of the cardboard, respectively to validate the entire measuring chain. The obtained results showed, however, that the cardboard reflection is not perfectly diffuse, having a fairly obvious directional component. This aspect can also be seen in Fig. 8 .

In Fig. 9 the luminance distribution $\left(\mathrm{cd} / \mathrm{m}^{2}\right)$ is indicated for 250 elements in the vector with results (abscissa). For the $5 \mathrm{~m}$ long (AB), i.e. a measurement density of $2 \mathrm{~cm}$. An important observation is necessary, the power of the punctiform lamp and the level of luminance on the test surface was chosen to make luminaires with two orders of magnitude higher than those in street lighting, so that measurements can be made in the presence of street lighting.

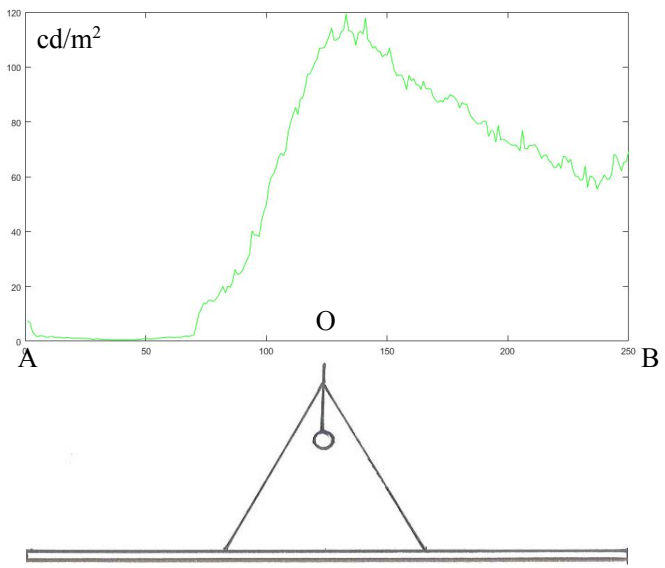

Fig. 10. Luminance distribution $\left(\mathrm{cd} / \mathrm{m}^{2}\right)$ for $\mathrm{AB}$ direction from Fig.7 and Fig.8.

Fig. 10 indicates that the cardboard reflection is not perfectly diffuse. Zone $\mathrm{AO}$, for which $\beta=180^{\circ}$ has a much lower reflection than zone $\mathrm{OB}$, for $\beta=0^{\circ}$. The results represent a demonstration of the method by which the luminance values of an area with a determined geometry can be extracted, by a single imaging measurement. From which then the reduced luminance coefficient could be calculated.

\section{CONCLUSIONS}

Street lighting is well regulated and benefits from a welldefined calculation algorithm, but a special parameter, reduced luminance coefficient has been shown to be not well enough known. Even if this parameter is standardized, the fact that it changes over time is not reflected in the design and especially in the operation of lighting systems. It has been shown that there is no mathematical relationship between reflection factor for diffuse reflection and Reduced Luminance Coefficient. Specular reflection being very far from diffuse reflection, and luminances must be calculated with relations closer to the calculation of the luminance of a direct source (or reflected in a mirror). Periodic measurement of this parameter, in the field, will allow the adaptive dimming of lighting systems, lower in the case of fresh asphalt and wider as the asphalt wears out and becomes more glossy. However, measuring Reduced Luminance Coefficient is difficult, based on laboratory equipment, such as a goniophotometer. The paper proposes an in-situ method for measuring Reduced Luminance Coefficient, based on an imaging method. Using a single point lighting source, the map of the luminances in the vicinity of the respective source is obtained, and based on the known geometry of the scene, the Reduced Luminance Coefficient is finally calculated.

\section{REFERENCES}

[1] L. Williams, "End of the asphalt road?", Engineering \& Technology, pp. 72-73, 2018.

[2] F. Duarte, F. Casimiro and D. Correia, "A new pavement energy harvest system," vol. International Renewable and Sustainable Energy Conference (IRSEC), 2013.

[3] C. Papadimitriou, C. Psomopoulos and F. Kehagia, "A review on the latest trend of Solar Pavements in Urban Environments," Energy Procedia 157 , Vols. Technologies and Materials for Renewable Energy, Environment and Sustainability, TMREES18, pp. 945-952, 2019. 
[4] A. Baumgartner and S. Amann, "Infrared reflectance factor of various asphalts," Remote Sensing for Agriculture, Ecosystems, And Hydrology Xx, no. DOI: 10.1117/12.2325509, 2019.

[5] V. Schomerus, D. Rosebrock and F. Wahl, "Camera-based Lane Border Detection in Arbitrarily Structured Environments," vol. IEEE Intelligent Vehicles Symposium (IV), 2014.

[6] A. Rusu, D. Lucache and C. Galatanu, "Calculation of energy economy by dimming led lamps with maximum performance," vol. 19th IEEE International Conference on Environment and Electrical Engineering (EEEIC) / 3rd IEEE Industrial and Commercial Power Systems Europe Conference (I\&CPS Europe), 2019.

[7] G. Zdunic, "Public lighting - road to energy efficiency," vol. 5th International Youth Conference on Energy (IYCE), 2015.

[8] L. Lipnický, D. Gašparovský and R. Dubnič, "Influence of the calculation grid density to the selected photometric parameters for road lighting," vol. IEEE Lighting Conference of the Visegrad Countries (Lumen V4), 2016.

[9] I. Ciobanu and R. Pentiuc, "Analysis on the possibility of using retrofit solutions for increasing the energy efficiency of public lighting systems," vol. International Conference on Applied and Theoretical Electricity (ICATE), 2016.

[10] A. Sedziwy and L. Kotulski, "A New Approach to Power Consumption Reduction of Street Lighting," International Conference on Smart Cities and Green ICT Systems (SMARTGREENS), 2015.

[11] I. Fryc , F. Bisegna and P. Tabaka, "Lighting of recreation grounds as a source of sky glow - The influence of luminaire type on this phenomenon," IEEE International Conference on Environment and Electrical Engineering and 2017 IEEE Industrial and Commercial Power Systems Europe (EEEIC / I\&CPS Europe), 2017.

[12] P. Tabaka and I. Fryc, "Landscape Lighting as a Source of Light Pollution - the Effect of the Seasons on this Phenomenon," IEEE Lighting Conference of the Visegrad Countries (Lumen V4), 2016.

[13] R. Bayer, M. Brejcha, Z. Pelánová and J. Zálešák, "Road lighting design by means of genetic algorithm," vol. 16th International Scientific Conference on Electric Power Engineering (EPE), 2015.
[14] L. Doulos, I. Sioutis, P. Kontaxis, G. Zissis and K. Faidas, "A decision support system for assessment of street lighting tenders based on energy performance indicators and environmental criteria: Overview, methodology and case study," Sustainable Cities and Society, vol. 51, 2019.

[15] ***, EN 13201, Road lighting - Part 3: Calculation of performance

[16] L. Doulos, I. Sioutis, A. Tsangrassoulis, L. Canale and K. Faidas, "Minimizing lighting consumption in existing tunnels using a no-cost fine-tuning method for switching lighting stages according revised luminance levels," vol. IEEE International Conference on Environment and Electrical Engineering and 2019 IEEE Industrial and Commercial Power Systems Europe (EEEIC / I\&CPS Europe), 2019.

[17] B. Li and L. Gu, "The development of LED street lamp pavement lighting effects testing system," 11th China International Forum on Solid State Lighting (SSLCHINA), 2014.

[18] A. Rusu, D. Lucache and G. Livint, "Assessment of Conflict Area Parameters Measurements by Using Photographic Methods," vol. International Conference on Electromechanical and Energy Systems (SIELMEN), 2019.

[19] J. Zhang, Y. Yang, X. He, M. Cheng, G. Cao and N. Zou, "Influence analysis of road reflective characteristic on roadway luminary design," vol. Proceedings of ICCTA2011, 2011.

[20] N. Tran, B. Powell, H. Marks, R. West and A. Kvasnak, "Strategies for Design and Construction of High-Reflectance Asphalt Pavements," Journal of the Transportation Research Board, pp. 124-130, 2009.

[21] E. Puttonen, J. Suomalainen, T. Hakala and J. Peltoniemi, "Measurement of Reflectance Properties of Asphalt Surfaces and Their Usability as Reference Targets for Aerial Photos," IEEE Transactions on Geoscience and Remote Sensing, vol. 47, 2009.

[22] S. Fotios and R. Gibbons, "Road lighting research for drivers and pedestrians: The basis of luminance and illuminance recommendations," Lighting Research\&Technology, vol. 50, p. 154$186,2018$. 